\title{
Calidad del fruto de aguacate con aplicaciones de ANA, boro, nitrógeno, sacarosa y anillado ${ }^{1}$
}

\author{
Avocado fruit quality with applications of ANA, boron, nitrogen, saccharose \\ and girdling
}

\author{
Joaquín Guillermo Ramírez-Gil ${ }^{2}$
}

\begin{abstract}
Resumen
El objetivo de este trabajo fue evaluar el efecto de la aplicación foliar de productos para el amarre, producción y calidad de los frutos de aguacate Hass. Esta investigación se realizó en el departamento de Antioquia, Colombia, en un lote de clima frío moderado. Se determinaron los factores de inducción de la floración, tipos de inflorescencia y alternancia de cosechas. Se realizó el anillado de ramas y aplicaciones foliares simple y combinada de ácido 1naftalenacético (ANA), boro, nitrógeno y sacarosa como fuente de energía. Se utilizó un diseño de bloques completos con cinco repeticiones y un árbol como unidad experimental, con tres evaluaciones (2010, 2011 y 2012). Se evaluó el amarre inicial y final, la cantidad y la calidad de la fruta producida. Los resultados indicaron que la inducción floral se presentó en periodos de menor precipitación con bajas temperaturas; las inflorescencias fueron en su mayoría indeterminadas, y se presentó una alternancia leve de producción. El amarre inicial del fruto se incrementó con el anillado y la aplicación de boro (28\%), mientras que el amarre final se favoreció con el anillado en 33\%; estos resultados no mejoraron la producción, característica que se incrementó con la adición de sacarosa sola y en combinación con boro, nitrógeno y ANA en un 14.5, 15.9, 14.9 y 14.4\%, respectivamente. La adición de sacarosa individual y combinada con la aplicación foliar de boro, nitrógeno y ANA, mejoraron la calidad y cantidad de fruta en la variedad Hass.
\end{abstract}

Palabras claves: producción de aguacate, desempeño de cultivos, incisión anular, aplicación foliar.

\begin{abstract}
The objective of this study was to evaluate the effect of foliar application of products for fruit set, production and quality of Hass avocado. This research was performed in a terrain that is located in a moderately cold weather, in Antioquia, Colombia. Factors for inducing flowering, types of inflorescence and production alternation were determined. In addition to the aforementioned, simple and combined foliar application of acid 1-naphthaleneacetic (ANA), boron, nitrogen, saccharose as source energy, and girding branches, was performed. For the experiment we used a complete block experimental design, with five replicates per treatment and a single tree as experimental unit, with three evaluations over time (2010, 2011 and 2012). The variables evaluated were initial and final fruit set, quantity, and quality of produced fruit. Results associated with this study indicate that floral induction in this variety occurs in periods of low rainfall and low temperatures, the inflorescences were mostly of an indeterminate type, and a slight alternation of production occurred. Initial fruit and final set increased with girdling branches and with the application of boron and girdling branches in $28 \%$ and $33 \%$ respectively, but this treatments did not improve the
\end{abstract}

\footnotetext{
1 Recibido: 12 de mayo, 2016. Aceptado: 14 de julio, 2016. Este trabajo formó parte de un proyecto de investigación financiado por la finca Piedras Blancas ubicada en el municipio de Donmatias, Antioquia, Colombia.

2 Universidad Nacional de Colombia, Facultad de Ciencias Agrarias, sede Medellín, Colombia. jgramireg@unal.edu.co
} 
production, instead the production was favored by adding saccharose alone, and in combination with boron, nitrogen and ANA, it presented increments of $14,5,15.9,14.9$ and $14.4 \%$ respectively. Results indicate that the addition of an individual external energy source combined with the foliar application of boron, nitrogen and ANA, improves the quality and quantity of fruit in the Hass avocado.

Keywords: avocado production, crop performance, girdling, foliar application.

\section{Introducción}

El cultivo de aguacate (Persea americana Mill) en Colombia está experimentando una alta dinámica, lo que ha conducido al incremento del área sembrada (Ramírez-Gil et al., 2014; Ramírez-Gil, 2016). Al ser Colombia un país que apenas empieza su experiencia con este frutal, presenta muchos retos para generar un paquete tecnológico propio de una producción sostenible, donde es necesario buscar el máximo potencial productivo de Persea americana Mill y sus variedades comerciales como el Hass. Mejores tecnologías de producción harán más competitivo este sistema productivo y así, se podrá aprovechar los tratados de libre comercio que ha firmado actualmente Colombia, donde este frutal representa una buena oportunidad (Ramírez-Gil, 2016).

Se denomina "amarre", como el tiempo y espacio en que el óvulo de la flor ha sido fecundado y el fruto inicia su formación (Rebolledo y Romero, 2011). En el cultivo de aguacate uno de los factores claves que determina en gran parte la capacidad productiva, es que a pesar del alto número de flores que puede producir, pocas son las que finalmente cuajan, y una mínima cantidad llega a frutos que se pueden cosechar (Tomer y Gottreich, 1978; Bergh, 1986; Whiley et al., 1988). Este fenómeno trae como resultado que el rendimiento mundial promedio solo sea cercano a $9 \mathrm{t} / \mathrm{ha}$ (FAO, 2015), muy inferior al potencial productivo de la especie que se cree pueda estar en 32,5 t/ ha (Wolstenholme, 1986; Gazit y Degani, 2007). Estudios desarrollados al respecto, buscando dar la explicación a este fenómeno, han dado como resultado la relación existente de múltiples factores asociados, en los que se destacan la competencia entre flujos de crecimiento vegetativo y reproductivo (Scholefield et al., 1985; Zilkah et al., 1987), el balance endógeno de hormonas como las giberelinas involucradas en los procesos de floración (Rossouw y Robbertse, 2001), el alto gasto energético para la producción del fruto (Wolstenholme, 1986), la participación de nutrientes como el boro y el nitrógeno (Jaganath y Lovatt, 1998), entre otros.

A nivel mundial se han realizado muchos estudios en los que se busca aumentar el amarre del fruto, además de mejorar su calidad, es así como se reportan muchas estrategias, las cuales no son concluyentes y sus resultados son muy variables. Dentro de las más comunes para este fin se encuentran las aplicaciones foliares del ácido giberélico $\left(\mathrm{AG}_{3}\right)$, nitrógeno $(\mathrm{N})$ y la práctica del anillado en ramas productivas (Espindola et al., 2008). Estos tratamientos han dado como resultado mejoras en muchas variables productivas de la especie. Para el caso del uso de la hormona $\mathrm{AG}_{3}$ se produjo el aumento del peso en frutos comerciales (Salazar-García y Lovatt, 2000). Por su parte, el efecto de las aplicaciones de nitrógeno se relacionó con un mayor desarrollo de las estructuras reproductivas de la planta, lo que se traduce en una mayor producción (Jaganath y Lovatt, 1998). Con respecto a la práctica del anillado, se reportan aumentos en producción y tamaño del futo cuando esta práctica se realiza en árboles productivos (Davie et al., 1995).

Dado el continuo crecimiento de este frutal, la poca investigación desarrollada en el país y las necesidades de aumentar los rendimientos por hectárea, este trabajo tuvo como objetivo evaluar el efecto de la aplicación foliar de productos para el amarre, producción y calidad de los frutos de aguacate cv Hass. 


\section{Materiales y métodos}

\section{Localización}

Este trabajo se desarrolló en un lote de 4 ha sembrado con aguacate variedad Hass injertado sobre un patrón de la raza antillana, establecido en un marco de plantación de 7 x $7 \mathrm{~m}$, de siete años de edad, ubicado en el municipio de Donmatías, Colombia $\left(6^{\circ} 29^{\prime} 54,19^{\circ} \mathrm{N}, 75^{\circ} 21^{\prime} 27^{\circ} \mathrm{O}-2379 \mathrm{~m}\right)$, cuya evaluación se desarrolló durante los años 2010 , 2011, 2012. El lote se encuentra ubicado a una altura de $2379 \mathrm{msnm}$, con $2500 \mathrm{~mm}$ de precipitación promedio anual y una temperatura promedio de $18^{\circ} \mathrm{C}$, clasificado como zona de vida bosque muy húmedo montano bajo bmh-MB (Holdridge, 1967). El lote experimental está ubicado sobre una posición geomorfológica de vertientes y colinas, relieve ondulado, pendiente del 5-40\%, drenaje natural, cuyo suelo presentó las siguientes características: textura franca (F), material parental de cuarzodioritas y cenizas volcánicas, suelo clasificado como Andic humitropept (AH 0-15, Abh 15-50, Bs 50-120, C $120+\mathrm{cm}$ ). Por su parte las características químicas fueron las siguientes: pH 5,4 (agua, 1:2, V:V); Al: 0,8 cmolc/kg (KCl 1M); Ca, Mg, y K: 1,6, 0,3, y 0,35 cmolc/kg (acetato de amonio $1 \mathrm{M}$ ); $\mathrm{Fe}, \mathrm{Mn}, \mathrm{Cu}$, y Zn: 237, 10, 5, y $3 \mathrm{mg} / \mathrm{kg}$ (Olsen-EDTA), B: 0,3 mg/kg (agua caliente); $\mathrm{S}: 12 \mathrm{mg} / \mathrm{kg}$ (fosfato de calcio $0,008 \mathrm{M}$ ); $\mathrm{NO}^{3-:}: 26 \mathrm{mg} / \mathrm{kg}$ (sulfato de aluminio 0,025 M); $\mathrm{NH}^{4+}: 33 \mathrm{mg} / \mathrm{kg}(\mathrm{KCl} \mathrm{1M}) ; \mathrm{P}: 8 \mathrm{mg} / \mathrm{kg}$ (Bray II) y contenido de materia orgánica del 10\% (Walkley y Black).

\section{Unidades experimentales}

La unidad experimental consistió de un árbol de siete años de edad, garantizando que para los tratamientos de aspersión foliar el 50\% de las flores estuvieran en estado de coliflor (Salazar-García et al., 1998) (Figura 1 A), y para la práctica del anillado en 50\% de floración desarrollada (Espindola et al., 2008). Todos los tratamientos fueron aplicados a toda la planta, dentro de estas se seleccionaron todas las ramas productoras y en cada rama se tomaron datos en mínimo tres inflorescencias. Con el fin de evitar cambios en las respuestas de las unidades experimentales en los tratamientos con respecto al control a todos los árboles, se realizó un manejo integrado de la fertilización, plagas y enfermedades durante todo el periodo de evaluación, según los criterios personales del asistente técnico de la finca.

\section{Tratamientos evaluados}

Se evaluaron dieciséis tratamientos y un control, con el fin de identificar cuales presentaban mejor efecto sobre la retención, cantidad y calidad de la fruta. T1: C1: control (aspersión con agua), T2: T.A: práctica de anillado ( $1 \mathrm{~cm}$ en el 50\% de las ramas), T3: T.B: aplicación foliar de boro (11 kg/ha de ácido bórico (10\% de boro)), en solución con agua destilada estéril, esta dosis se ajustó a cada uno de los árboles seleccionados para el tratamiento (42,3 g/planta de ácido bórico (10\% de boro)), con base en una densidad de siembra de 260 plantas/ha. T4:T.ANA: aplicación foliar de ANA (25 mg/l de ácido 1-naftalenacético, en solución con agua destilada estéril); T5: T.C: aplicación foliar de una fuente de energía (50 g/árbol de sacarosa, en solución con agua destilada estéril), T6: T.N: aplicación de nitrógeno (160 g/árbol de carbamida (urea al 46\%)), en solución con agua destilada estéril), T7: T. B+T.ANA; T8: T.B+T.C, T9: T.B+T.N, T10: T.B+T.A, T11: T.ANA+T.C, T12: T.ANA+T.N, T13: T.ANA+T.A; T14: T.C+ T.N; T15: T.C+T.A y T16: T.N+T.A.

Para los tratamientos en donde se utilizó la aplicación de nitrógeno y el anillado, el procedimiento se realizó según las indicaciones de Espindola et al. (2008). Por su parte, lo relacionado con el nutriente boro, la dosis, la forma 

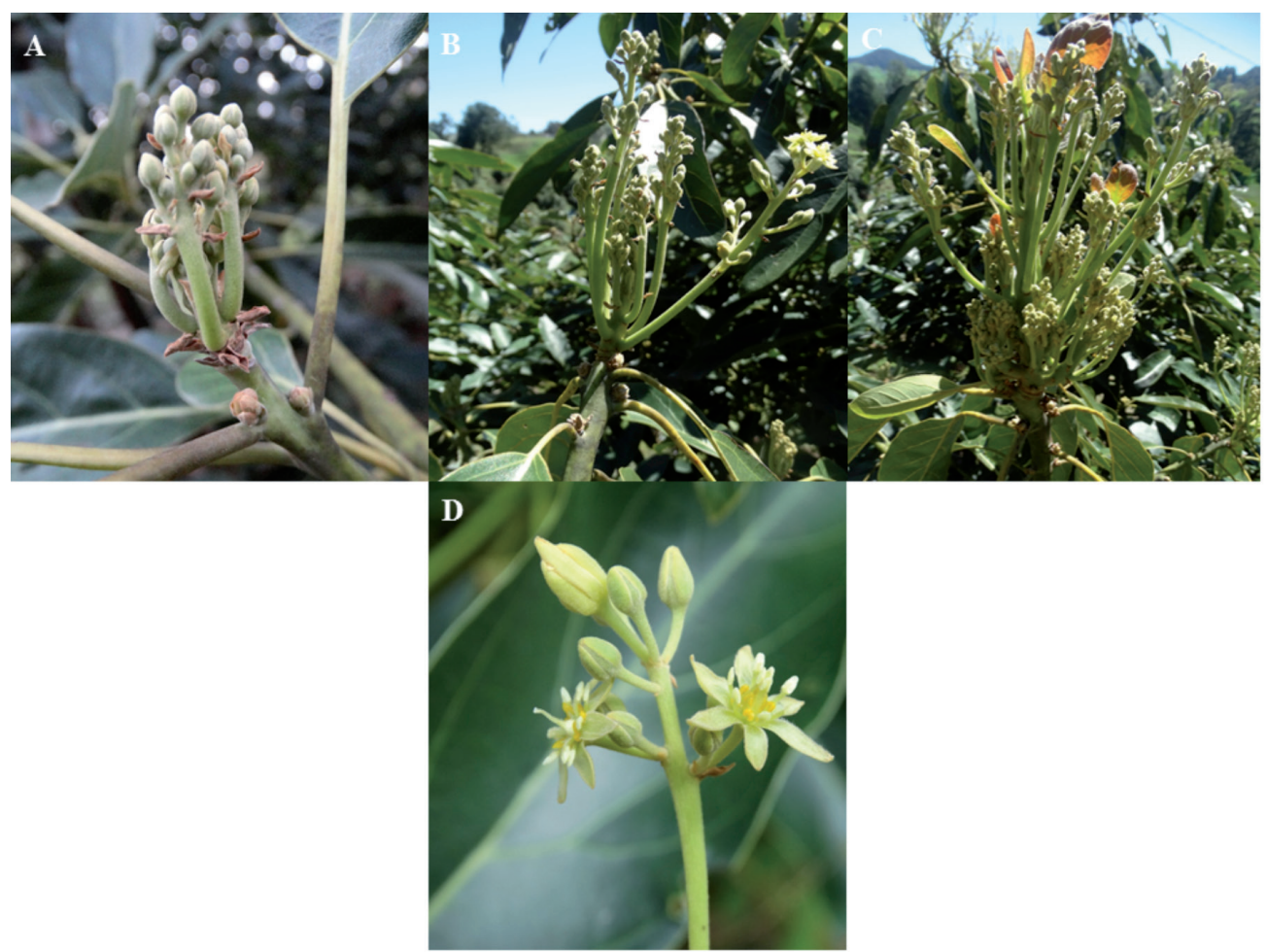

Figura 1. Registro fotográfico de algunas variables morfológicas asociadas con la fisiología de $P$. americana cv Hass, bajo condiciones en Donmatias, Antioquia, Colombia, durante los años 2010,2011 y 2012.

A: inflorescencia en estado de Coliflor. B: inflorescencia de tipo determinada. C: inflorescencia de tipo indeterminada. D: Flor abierta de tipo masculino (derecha) y flor abierta de tipo femenino (izquierda).

Figure 1. Photographic record of some morphological variables associated with physiology of $P$. americana Hass under field conditions in Donmatias, Antioquia, Colombia, during the years 2010, 2011 and 2012.

A: Cauliflower state inflorescence. B: inflorescence determined type. C: inflorescence undetermined type. D: open male flower type (right) and open female flower type (left).

y el momento de aplicación se realizaron siguiendo las especificaciones de Li et al. (1997). Para la determinación de la cantidad y el tipo de fuente de energía se siguió la recomendación de Ramírez-Gil et al. (2013). Todos los tratamientos en los cuales se realizó la aspersión foliar, fueron aplicados a toda la planta o unidad experimental, mediante aspersión manual (1,5 Psi) con boquillas de abanico plano; la cantidad de agua destilada estéril fue la necesaria para cubrir toda el área foliar. Con excepción del anillado, en todos los tratamientos se realizaron tres aspersiones por ciclo, el nitrógeno y el boro se aplicaron durante la fase de floración, mientras que la aplicación de la fuente de energía, se realizó una en la fase de floración y otra en la etapa de llenado del fruto.

\section{Variables determinadas}

En el lote experimental se evaluaron aspectos generales asociados a la floración y producción del aguacate cv Hass, dentro de estos se determinaron los meses del año asociados a la floración y su relación con variables 
climáticas como la precipitación, temperatura diurna y nocturna, variables determinadas en una estación climática (WatchDog serie 2000). Además, se cuantificó el tipo de inflorescencia (determinadas e indeterminadas), la dicogamia floral y la producción acumulada por año, con el fin de establecer si había presencia de alternancia en cosecha. Los tratamientos fueron aplicados en las unidades experimentales (un árbol en producción), pero las variables se determinaron sobre las ramas e inflorescencias marcadas. Las variables de respuesta determinadas fueron las sugeridas por Espindola et al. (2008), con algunos cambios, dando lugar a los siguientes parámetros: \% de amarre inicial ((número de frutos amarrados en el tiempo 1/número de flores) *100, frutos con diámetro $>0,5$ $\mathrm{cm}$ ), $\%$ amarre final ((número de frutos amarrados en el tiempo 2 /número de flores *100), frutos con diámetro $>10$ $\mathrm{cm}$ ). Además, se complementó con la producción acumulada (obtenida como la cantidad total por planta/ciclo) y calidad (extra e industrial), según las indicaciones de Ramírez-Gil et al. (2017).

\section{Diseño y análisis estadístico}

Se utilizó un diseño en bloques completos al azar, donde el criterio de bloqueo fue la pendiente del terreno (0$10,10,1-20,20,1-40,40,1-60$ y $>60,1 \%$ ), con cinco repeticiones por tratamiento. Las evaluaciones se desarrollaron en los años 2010, 2011 y 2012. La homocedasticidad y normalidad de los datos fue analizada $(\mathrm{P}<0,05)$ con base en los criterios de Levene (Levene, 1960) y Kolmogorov-Smirnov (Massey, 1951) respectivamente; posteriormente, se realizó el análisis de varianza y prueba de comparación de medias de Tukey con un nivel de significancia del $95 \%$. También se realizó un análisis de correlación múltiple, con el fin de determinar la asociación entre el amarre inicial y final, y la cantidad y calidad de la fruta, cuyos parámetros estadísticos fueron el coeficiente de correlación $\left(\mathrm{r}^{2}\right)$ y su significancia $(\mathrm{P}<0,05)$. Los cálculos se realizaron mediante el programa computacional $\mathrm{R}$ (R-Core-Team, 2013).

\section{Resultados y discusión}

\section{Aspectos generales asociados a la floración y producción}

Bajo las condiciones edafoclimáticas del lote evaluado (Figura 2A), las plantas de aguacate cv Hass, exhibieron dos floraciones por año; la primera se presentó en los meses de noviembre-enero, representando el $70 \%$ del total de las floraciones del año, con enero como plato del evento fisiológico. El 30\% restante de la floración ocurrió en los meses de julio-agosto, donde para julio el valor fue del 18\%, y de 12\% para agosto. La diferenciación floral, y dentro de ella la aparición de los estadíos 1 hasta el 9 (Salazar-García et al., 1998), coincidió con la menor precipitación ocurrida en estos meses, la cual se presentó en los mismos periodos donde hubo disminución de la temperatura, hasta presentarse valores inferiores a $8{ }^{\circ} \mathrm{C}$ (Figura 2A). Durante el ciclo floral se presentó la fase normal del grupo A, donde las flores abrieron como femeninas por la mañana y masculinas en la tarde del día siguiente, pero se observó que en algunos días había un traslape de aproximadamente una hora, en el cual se podían encontrar flores femeninas y masculinas abiertas, estos traslapes coincidieron con cambios fuertes en la temperatura y humedad ambiental (Figura 1D). Los resultados obtenidos concuerdan con lo reportado por Loupassaki et al. (1995) y Gazit y Degani (2007). Por su parte las inflorescencias fueron del tipo determinada e indeterminada (Figura 1B y C), presentándose una mayor cantidad de estas últimas (Figura 2B).

Se considera que la inducción floral en aguacate en las zonas tropicales se da por consecuencia del estrés hídrico (Wilkie et al., 2008), mientras que para las zonas subtropicales ocurre por la disminución de la temperatura (Salazar-García y Lovatt, 2002). Para Colombia no se tienen actualmente datos sobre el comportamiento floral de la variedad Hass, pero en la variedad Lorena se ha encontrado que la aparición de los estadíos A (yema en latencia), B (abandono de reposo), D (desarrollo de la inflorescencia) y F (antesis) presentaron una relación significativa con la 

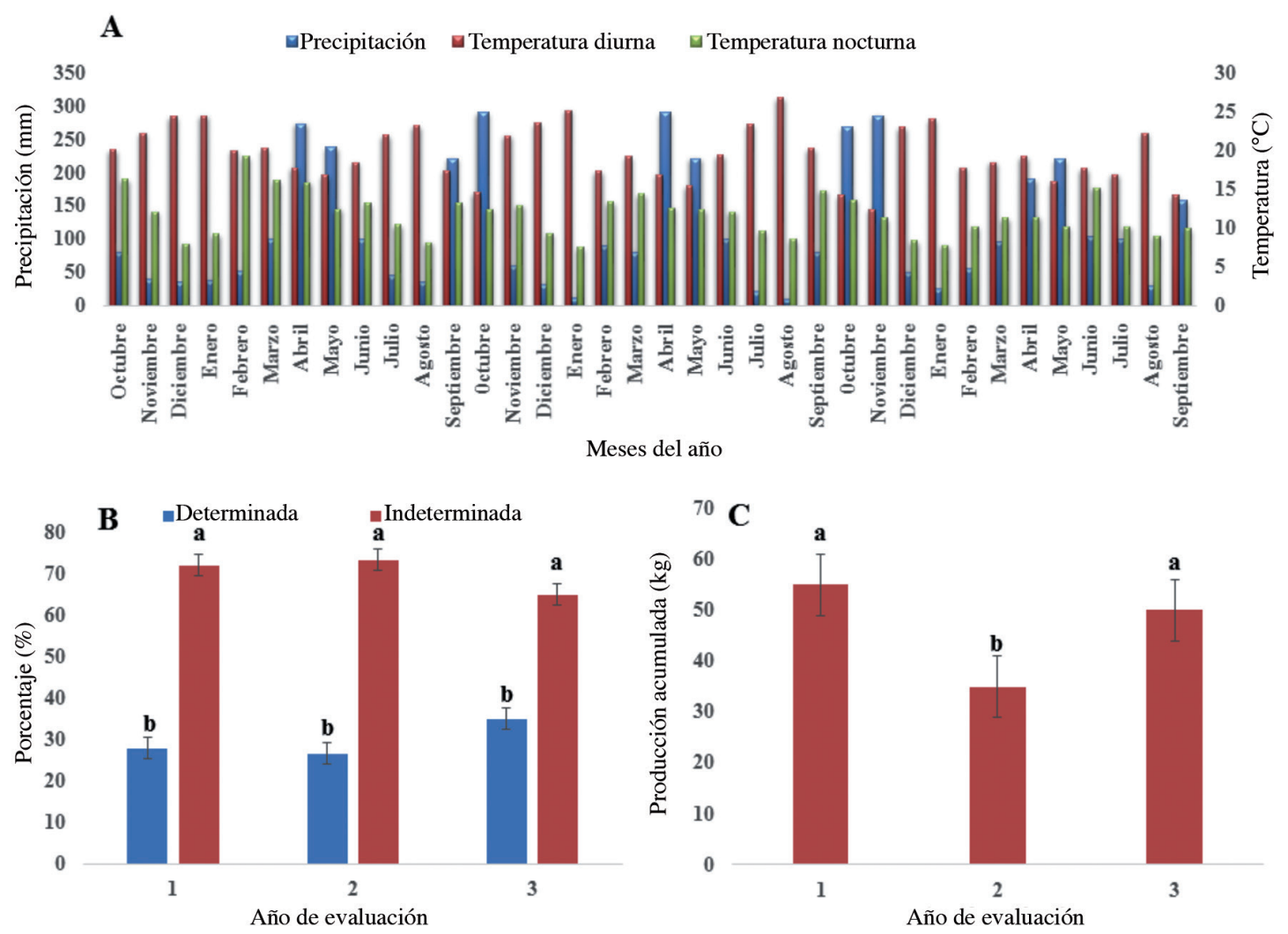

Figura 2. Aspectos básicos asociados con la fisiología de $P$. americana cv Hass bajo condiciones en Donmatias, Antioquia, Colombia, durante los años 2010, 2011 y 2012.

A: variables climáticas asociadas a los años 2010, 2011 y 2012 en el lote experimental. B: relación entre los tipos de influencias para los años 2010 (1), 2011 (2) y 2012 (3). C: producción acumulada para los años 2010 (1), 2011 (2) y 2012 (3). Letras distintas significan diferencias significativas $(\mathrm{P}<0,05)$.

Figure 2. Basic aspects associated with physiology of $P$. americana $\mathrm{cv}$ under field conditions in Donmatias, Antioquia, Colombia, during the years 2010, 2011, and 2012.

A: climatic variables associated with the years 2010, 2011, and 2012 in an experimental lot. B: relationship between the types of influences for 2010 (1), 2011 (2), and 2012 (3). C: cumulative production for 2010 (1), 2011 (2), and 2012 (3). Different letters mean significant differences $(\mathrm{P}<0,05)$.

precipitación y la humedad relativa durante el período de evaluación (Romero, 2011). Basado en lo encontrado en este trabajo es posible que los dos factores estén involucrados, pero es necesario realizar estudios en otras regiones del país para determinar con exactitud los determinantes asociados a la inducción floral para la variedad Hass.

En lo que respecta a la producción acumulada en el tiempo, se evidenció la alternancia de cosechas, dado que para el año 2010, la producción fue superior $(\mathrm{P}<0,05)$ con respecto a la del año siguiente, mientras que para el 2012 incrementó nuevamente (Figura 2C). Esta disminución encontrada en el año 2011 equivalió a 37\% de la producción por árbol con respecto al año anterior, y de 30\% con el año posterior, estos cambios fueron altamente variables, dado que se presentaron hasta nivel de árbol en la finca. El fenómeno de alternancia ha sido reportado en sistemas de producción de aguacate, el cual se caracteriza por una elevada carga de cosecha en un ciclo de producción (año “on”) y una baja carga de cosecha en el siguiente ciclo (año "off”) (Salazar-García et al., 1998; Dixon, 2007). Para las condiciones de Colombia este fenómeno fue reportado en la variedad Lorena en dos años de producción continua (Romero, 2011). 


\title{
Alternativas para el amarre y calidad de la fruta
}

\author{
Amarre inicial y final de frutos
}

El porcentaje de amarre inicial en los tratamientos evaluados presentó diferencias estadísticamente significativas $(\mathrm{P}<0,05)$, donde los valores más altos se encontraron en los tratamientos combinados de la práctica del anillado y la aspersión foliar de boro, nitrógeno y la fuente de energía (T.A+T.B, T.A+T.N y T.A+T.C). En un segundo grupo se ubicaron las estrategias en las cuales se utilizó la práctica del anillado individual y combinado con el ANA, aplicación foliar de boro y su combinación con nitrógeno, la fuente de energía y ANA, y la combinación de estas (T.A, T.A+T.ANA, T.B+T.N, T.B+T.C, T.B+T.ANA, T.N+T.ANA y T.C+T.ANA). En un tercer grupo se ubicaron los tratamientos en los cuales se realizó la aplicación foliar de boro, nitrógeno y la fuente de energía en forma individual (T.B, T.N y T.C). Mientras que en el nivel más bajo se ubicó la aplicación individual de ANA y el control (C1) (Figura $3 \mathrm{~A}$ ).

Los tratamientos con valores superiores como T.A+T.B, T.A+T.N y T.A+T.C, lograron un amarre inicial de 10 , 9,8 y $9,5 \%$, lo que equivalió a incrementos del 28,26 y $25 \%$, respectivamente, con respecto al control (C1). Con la práctica del anillado el valor incrementado fue de $20 \%$, el mayor en todas las estrategias evaluadas de manera individual. Al respecto, Espindola et al. (2008) reportaron que la aplicación de nitrógeno y el anillado lograron aumentos de amarre inicial muy superiores a los encontrados en este trabajo, los cuales fueron del 50 y $91,6 \%$ en el año "off" y del 26,6 y 23,6\% en el año "on", pero contrario a lo encontrado en este trabajo no reportaron efecto de las interacciones en el primer año de evaluación, pero si en el siguiente con incrementos en el amarre inicial del 23,8 y $13,6 \%$.

En lo que respecta al amarre final, los resultados conservaron parte de la tendencia encontrada en el amarre inicial, dando lugar a una correlación positiva $(\mathrm{P}<0,05)$ (Cuadro 1). En estos se encontró que los tratamientos con los valores más altos $(\mathrm{P}<0,05)$ fueron aquellos en los cuales se realizó la combinación del anillado con las aspersiones de la fuente de energía (T.A+TC) y el anillado individual (T.A), con incrementos del 30,7 y $33 \%$ respecto al control (C1). En un lugar intermedio $(\mathrm{P}<0,05)$ se ubicaron los tratamientos en donde se utilizó individualmente la aspersión de la fuente de energía (T.C), la combinación del anillado con el boro (T.A+T.B), el boro con la fuente de energía (T.B+T.C) y el nitrógeno con la fuente de energía (T.N+T.C), los cuales incrementaron el amarre final en 23,7, 22,4, 25, 25, 22,2, y $27,4 \%$, respectivamente. En un tercer grupo $(\mathrm{P}<0,05)$ se ubicaron las adiciones individuales de boro, nitrógeno (T.B, T.N) y la combinación del tratamiento con el anillado y la hormona ANA (T.A+T.ANA), el nitrógeno con la hormona ANA (T.N+T.ANA), y la fuente de energía con la hormona ANA (T.C+T.ANA), con incrementos del 18,1, 19,6, 18,15 , y $16,6 \%$, respectivamente. Con respecto al resto de tratamientos, no se encontraron diferencias significativas $(\mathrm{P}>0,05)$ con el control (C1) (Figura 3B). Estos resultados indican que existen tratamientos en los cuales se logró un efecto estadísticamente significativo $(\mathrm{P}<0,05)$ en el aumento del amarre inicial y final de frutos, mismos que pueden actuar de forma individual, como el anillado, o de manera conjunta como las aspersiones de boro, nitrógeno y carbohidratos, indicando un efecto sinérgico entre ellos.

Los valores encontrados en este trabajo se relacionan con lo reportado por Espindola et al. (2008), los cuales reportaron aumentos de $41 \%$ en el amarre final cuando se realizó la práctica del anillado en los años de alta producción, y para el año de baja producción el anillado y aplicación de nitrógeno registraron aumentos del 88,8 y $25 \%$. Para la variable amarre final, los valores registrados en este trabajo fueron superiores a los reportados, ya que bajo las condiciones de este experimento el valor más alto fue del 3,5\%, comparado con el 2,4\% reportado como máximo por Espindola et al. (2008).

La práctica del anillado ha sido ampliamente reportada como una estrategia para un mejor amarre del fruto en aguacate. Se considera que su efecto se da porque interrumpe temporalmente el transporte de carbohidratos, metabolitos y algunas hormonas a la raíz, dando lugar a la acumulación en las ramas productoras (Davie et al., 

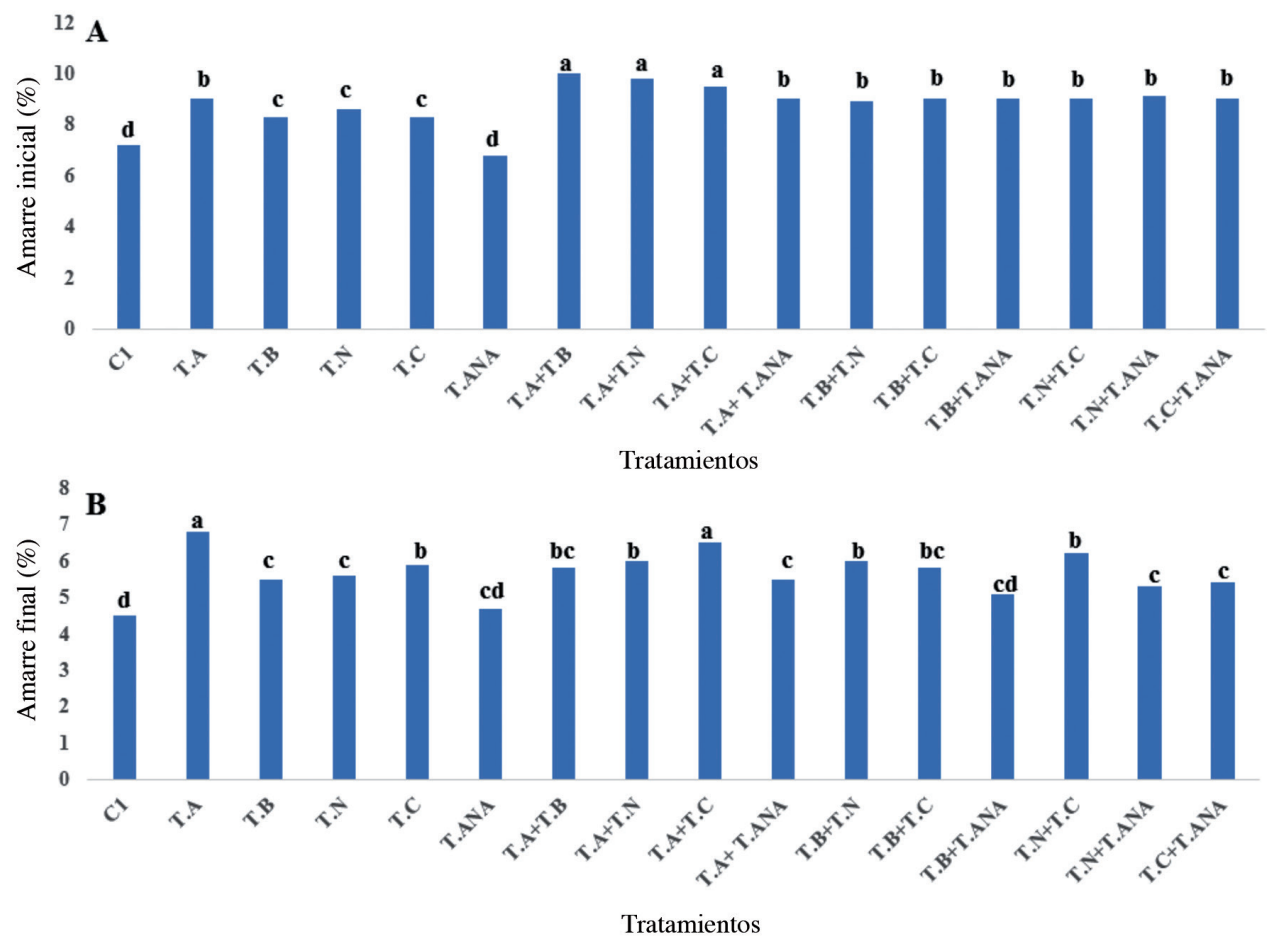

Figura 3. Amarre inicial y final de frutos de P. americana cv Hass asociados a distintos tratamientos bajo condiciones en Donmatias, Antioquia, Colombia, durante los años 2010, 2011 y 2012.

A: amarre inicial. B: amarre final. Letras distintas significan diferencias significativas $(\mathrm{P}<0,05)$. T: tratamiento. C1: control. T.A: anillado de ramas. T.B: aplicación foliar de boro. T.N: aplicación foliar de nitrógeno. TC: aplicación foliar de sacarosa. T.ANA: aplicación foliar de ácido 1naftalenacético.

Figure 3. Initial and final fruit set of P. americana cv Hass associated with different treatments under field conditions in Donmatias, Antioquia, Colombia, during the years 2010, 2011, and 2012.

A: Initial fruit set B: Final fruit set. Different letters mean significant differences $(\mathrm{P}<0.05)$. T: treatment. C1: control. T.A: girding branches. T.B: foliar application of boron. T.N: foliar application of nitrogen. TC: foliar application of saccharose. T.ANA: foliar application of acid 1- Naphthaleneacetic.

1995; Rebolledo y Romero, 2011). Además, se le ha atribuido el efecto de incrementar la velocidad de crecimiento del tubo polínico y penetración en el óvulo (Tomer y Gottreich, 1978). Por su parte, la aplicación de boro y nitrógeno han sido reportadas como estrategias que mejoran el amarre de los frutos en cultivos de aguacate (Abou et al., 1975; Zilkah et al., 1987; Dixon et al., 2005). La respuesta a la aplicación del boro depende de muchos aspectos, dentro de los cuales se encuentra la variedad y las dosis (Li et al., 1997).

El efecto de la aplicación de nitrógeno en el amarre de frutos se debe a la acción de este nutriente sobre el incremento de óvulos viables y número de tubos polínicos que alcanzan exitosamente el óvulo (Jaganath y Lovatt, 1998). Por su parte, el boro es esencial para los procesos como la germinación del polen, desarrollo del tubo polínico a través del estigma, estilo y ovario hasta alcanzar el óvulo, y para las divisiones mitóticas necesarias para producir el esperma (Lovatt y Dugger, 1984). 
Cuadro 1. Análisis de correlación múltiple entre las variables amarre inicial, final, producción acumulada, calidad extra y calidad industrial de frutos de P. americana cv Hass bajo condiciones en Donmatias, Antioquia, Colombia, durante los años 2010, 2011 y 2012.

Table 1. Analysis of multiple correlations between variables on the initial and final fruit set cumulative production, extra quality, and industrial quality under field conditions in Donmatias, Antioquia, Colombia, during the years 2010, 2011 and 2012.

\begin{tabular}{lccccc}
\hline & $\begin{array}{c}\text { Amarre inicial } \\
(\boldsymbol{\%})\end{array}$ & $\begin{array}{c}\text { Amarre final } \\
(\boldsymbol{\%})\end{array}$ & $\begin{array}{c}\text { Producción } \\
\text { acumulada }\end{array}$ & Extra & Industrial \\
\hline Amarre inicial (\%) & & ${ }^{2} 0,7635$ & $-0,0175$ & $-0,3315$ & 0,5154 \\
Amarre final (\%) & 0,7635 & & 0,9469 & 0,1936 & 0,0342 \\
& 0,0004 & & $-0,4291$ & $-0,6828$ & 0,8459 \\
Producción acumulada & $-0,0175$ & $-0,4291$ & 0,0856 & 0,0025 & 0,0000 \\
& 0,9469 & 0,0856 & & 0,9079 & $-0,6597$ \\
Extra & $-0,3315$ & $-0,6828$ & 0,9079 & 0,0000 & 0,0040 \\
& 0,1936 & 0,0025 & 0,0000 & & $-0,8781$ \\
Industrial & 0,5154 & 0,8459 & $-0,6597$ & $-0,8781$ & 0,0000 \\
& 0,0342 & 0,0000 & 0,0040 & 0,0000 & \\
\hline
\end{tabular}

${ }^{a}$ correlación existente entre las variables. ${ }^{\mathrm{b}} \mathrm{P}$-value, valores de P- value inferiores a 0,05 indican correlaciones estadísticamente significativas en el nivel de confianza del $95,0 \% /{ }^{a}$ correlation between variables. ${ }^{b} \mathrm{P}$-value, values below $0.05 \mathrm{P}$ - value indicate statistically significant correlations in a confidence level of a $95,0 \%$.

\section{Producción acumulada y calidad}

\section{Producción acumulada}

Los tratamientos que presentaron los valores más altos de producción acumulada $(\mathrm{P}<0,05)$, fueron aquellos en donde se realizó la aplicación foliar de una fuente de energía de manera individual y combinada con el uso de boro, nitrógeno y la hormona ANA (T.C, T.B+T.C, T.N+T.C y T.C+T.ANA) (Figura 4). Estos valores representaron incrementos en producción de 13,14, 12 y 13\%, respectivamente, con respecto al control (C1). Todos los tratamientos en que se utilizó el anillado como práctica individual o combinada con las demás estrategias (T.A. T.A+T.B, T.A+T.N, T.A+T.C y T.A+T.ANA), la producción acumulada fue inferior $(\mathrm{P}<0,05)$, comparado con el control (C1), dando lugar a pérdidas del 18, 14, 9, 7 y 6\%, respectivamente (Figura 4).

En lo que respecta a la categoría de tipo extra, correlacionó positivamente con la producción acumulada $(\mathrm{P}<0,05)$, dado que los valores más altos de esta variable estuvieron asociados con los tratamientos en los que la cantidad total de fruta cosechada fue mayor (Cuadro 1 y Figura 4). Mientras que la calidad industrial se correlacionó negativamente con la producción acumulada $(\mathrm{P}<0,05)$ (Cuadro 1 y Figura 4$)$. En este sentido, la calidad extra se correlacionó negativamente $(\mathrm{P}<0,05)$ con la industrial, dando lugar a que en los tratamientos con cantidades bajas de la primera, los niveles de la segunda fueron superiores (Cuadro 1 y Figura 4). Los resultados obtenidos en este trabajo sugieren que no existió correlación $(\mathrm{P}>0,05)$ entre los valores de amarre inicial y final, y la producción acumulada total (Cuadro 1), dado que los valores más altos de amarre inicial y final, no coincidieron con una mayor producción. Por su parte, estas variables sí presentaron correlación $(\mathrm{P}<0,05)$ con la calidad de la fruta, donde los valores más altos de amarre inicial y final, coincidieron con una mayor cantidad de la categoría industrial y una menor proporción de calidad extra (Cuadro 1). 


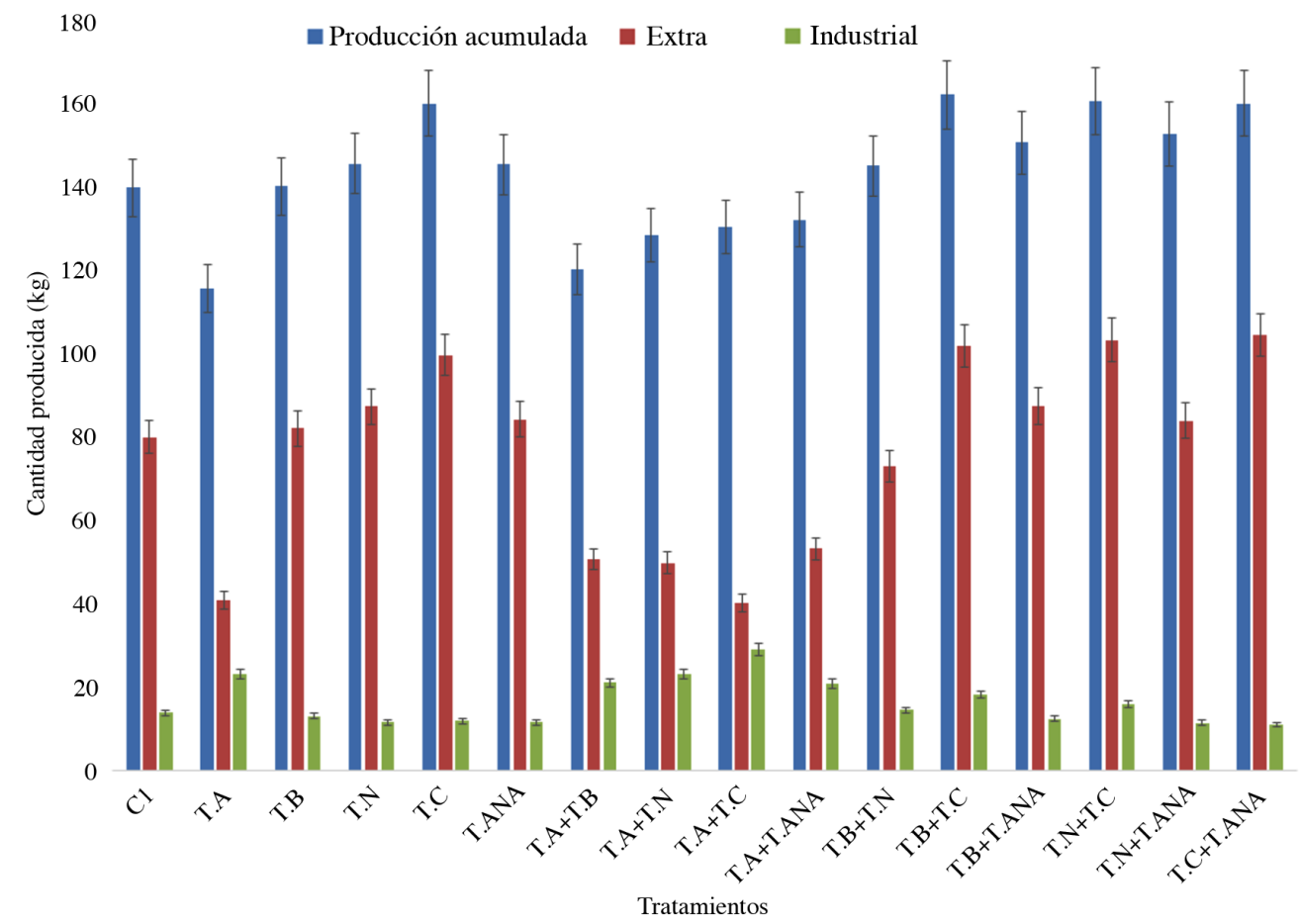

Figura 4. Producción acumulada y calidad de fruta de P. americana cv Hass asociados a aplicaciones de ANA, boro, nitrógeno, sacarosa como fuente de energía y anillado de ramas y bajo condiciones en Donmatias, Antioquia, Colombia, durante los años 2010, 2011 y 2012. Las barras en las figuras representan el intervalo de confianza de las medias, donde la superposición de estas indica que no hay diferencias significativas $(\mathrm{P}>0,05)$.

T: tratamiento. C1: control. T.A: anillado de ramas. T.B: aplicación foliar de boro. T.N: aplicación foliar de nitrógeno. TC: aplicación foliar de sacarosa. T.ANA: aplicación foliar de ácido 1naftalenacético.

Figure 4. Cumulative production and quality of fruits in P. americana cv Hass associated to foliar application of ANA, boron, nitrogen, sucrose as an energy source, and girdling branches under field conditions in Donmatias, Antioquia, Colombia, during the years 2010, 2011, and 2012. The bars in the figures represent the confidence interval of the means, where the overlap of the bars indicate that there are no significant differences $(\mathrm{P}>0.05)$.

T: treatment. C1: control. T.A: girding branches. T.B: foliar application of boron. T.N: foliar application of nitrogen. TC: foliar application of saccharose. T.ANA: foliar application of acid 1- Naphthaleneacetic.

Los tratamientos con mayor calidad extra $(\mathrm{P}<0,05)$ fueron aquellos en los que se realizó la aspersión foliar de una fuente de energía (T.C) y su combinación con la aspersión de boro, nitrógeno y ANA (T.B+T.C, T.N+T.C y T.C+T.ANA), los cuales lograron aumentos de 20, 22, 23 y 24\%, respectivamente, con respecto al control (C1); además, estos tratamientos lograron valores de calidad industrial inferiores $(\mathrm{P}<0,05)$. Los tratamientos en los cuales se realizó la práctica del anillado en las ramas (T.A, T.A+T.B, T.A+T.N, T.A+T.C y T.A+T.ANA) la calidad extra fue inferior $(\mathrm{P}<0,05)$, con respecto a los demás tratamientos (Figura 4), estas reducciones fueron del 48, 34, 36, 49 y $31 \%$ con respecto al control (C1). Estos resultados no concuerdan con los encontrados en otro estudio donde se reportaron aumentos en la cantidad producida y el tamaño hasta del 35\% con el uso del anillado en las ramas (Davie et al., 1995). 
En términos de productividad se encontró que la práctica que aumentó los rendimientos en cantidad y calidad, fueron las adiciones de una fuente de energía, la cual presentó efecto como estrategia individual o combinada con la aspersión de nitrógeno y boro. Este efecto podría estar asociado a que esta fuente energética externa puede suplir, en parte, las necesidades de la planta en los periodos de alta demanda como lo es el amarre y llenado de la fruta, disminuyendo la competencia entre los órganos reproductivos y vegetativos (Blumenfield et al., 1983). Una de las causas de los bajos rendimientos en el aguacate, se debe a que esta especie demanda grandes cantidades energéticas, para alcanzar los niveles de aceite en las distintas estructuras del fruto y las reservas asociadas a las semillas (Wolstenholme, 1987). El estudio de la dinámica de las fuentes de energía en aguacate, mostró que los niveles más bajos de carbohidratos se producen durante la floración, el crecimiento de brotes y desarrollo del fruto (Scholefield et al., 1985)

En lo que respecta al ANA, no influyó sobre el amarre inicial o final y la producción acumulada como estrategia individual o combinada con el boro y el nitrógeno, pero sí cuando se combinó con la fuente de energía. Sin embargo, las moléculas asociadas a la hormona ANA han presentado efectos positivos en manzanos, al ayudar en el raleo de flores, conduciendo a una producción más homogénea y comercial (Webster y Spencer, 1999).

Muchas otras prácticas agronómicas se han desarrollado y evaluado con el fin de aumentar la producción del aguacate, las cuales hasta el momento no son concluyentes. Dentro de las más estudiadas han sido las aplicaciones foliares de reguladores de crecimiento, en especial las giberelinas, con el fin de reducir la alternancia de la producción año tras año y mejorar la calidad de fruta, al lograr una compensación en los brotes vegetativos y productivos (Salazar-García y Lovatt, 2002). Por otra parte, se ha reportado que la reducción manual de frutos pequeños en huertos comerciales han dado resultados prometedores (Wolstenholme, 2010).

Las prácticas asociadas a la retención de fruta, no deben alterar la calidad y cantidad de esta, ya que algunas estrategias utilizadas para este fin pueden dar lugar a incrementos en un año en detrimento del otro. Esta situación se ha asociado a la práctica del anillado, lo cual se da como resultado de la alta demanda energética en el año con producción mayor, conduciendo a un aumento de los brotes vegetativos del año siguiente, con su efecto directo sobre la reducción de los brotes florales (Lovatt, 2006).

\section{Conclusiones}

La productividad de aguacate no necesariamente está relacionado con la cantidad de frutos que el árbol puede amarrar, ya que bajo los tratamientos en los cuales se presentaron bajos porcentajes de amarre inicial y final, la producción acumulada fue superior, además de que bajo estos tratamientos los porcentajes de calidad extra fueron mayores, indicando que una mayor retención da lugar a una mayor cantidad de frutos, pero de tamaño más pequeño, lo cual conduce a que la calidad y el peso acumulado sea menor. Por tal motivo, se asume que la baja producción del aguacate es el resultado de una serie de eventos que ocurren a lo largo del desarrollo reproductivo, donde puede presentarse un aumento en la abscisión de flores y frutos como resultado de numerosos factores incluyendo temperaturas extremas, deficiencias nutricionales y factores genéticos (Bower et al., 1990; Rebolledo y Romero, 2011). Por otra parte, se puede indicar que sí se decide utilizar una estrategia para la retención de fruta, esta debe ir acompañada de un plan de manejo integrado del sistema de producción con el fin de dar las condiciones más óptimas de desarrollo de la planta, para que pueda soportar el aumento en la producción sin afectar la calidad representada por el calibre y la producción de año siguiente.

\section{Agradecimientos}

En este trabajo se quiere agradecer al productor Carlos Noreña por la disponibilidad del lote de evaluación, la financiación y el mantenimiento de los ensayos montados durante los tres años que duró el proceso. 


\section{Literatura citada}

Abou, A., I. Desouki, and M. El-Tanahy. 1975. Effect of nitrogen fertilization on yield and fruit oil content of avocado trees. Sci. Hort. 3:89-94.

Bergh, B.O. 1986. Persea americana. In: A.B. Halevy, editor, CRC handbook of flowering. Vol. 5. CRC Press, Boca Raton, FL, USA. p. 253-268.

Bower, J., C. Lovatt, J. Cutting, and M. Blanke. 1990. Interaction of plant growth regulator and carbohydrate in flowering and fruit set. Acta Hort. 275:425-434.

Blumenfeld, A., S. Gazit, and E. Argaman. 1983. Factors involved in avocado productivity. Volcani Center, ISR.

Davie, S., P. Stassen, M. Van-Der-Walt, and B. Snijder. 1995. Girdling avocado trees for improved production. SAAGA 18:51-53.

Dixon, J. 2007. Shoot growth of 'Hass' avocado trees in 'on' and 'off' flowering years in the western bay of plenty. NZ Avocado Growers Association Ann. Res. Report 7:41-48.

Dixon, J., D. Smith, T. Elmsly, and F. Fields. 2005. The impact of foliar applications of nitrogen and boron on 'Hass' avocado fruit set in 2004. NZ Avocado Growers' Association Ann. Res. Report 5:27-34.

Espindola, M., R. Cano, J. Rodríguez, y P. Sánchez. 2008. Amarre de fruto en aguacate 'Hass' con aplicaciones de AG $_{3}$, N y anillado. Agric. Téc. Méx. 34:407-419.

FAO. 2015. Statistics. FAO Statistics Division, FAOSTAT. http://faostat3.fao.org/download/Q/QC/E. (accessed 20 nov. 2015).

Gazit, S., y C. Degani. 2007. Biología reproductiva. En: A. Whiley et al., editores, El palto, botánica, producción y usos. Ediciones Universitarias de Valparaíso, CHI. p. 103-131.

Holdridge, L. 1967. Life zone ecology. Tropical Science Center, San José, CRC.

Jaganath, I., and C.J. Lovatt. 1998. Efficacy studies on prebloom canopy applications of boron and/or urea to 'Hass' avocado. Acta Hort. 1:181-184.

Levene, H. 1960. Robust tests for the equality of variance. In: I. Olkin, editor, Contributions to probability and statistics: Essays in honor of Harold Hotelling. Stanford University Press, Palo Alto, CA, USA. p. 278-292.

Li, Y., J. Crane, T. Davenport, and C. Balerdi. 1997. Preliminary findings on the effects of foliar-applied urea and boron on plant nutrition, fruit set and yield of avocado trees. Proc. Fla. State Hort. Soc. 110:136-138.

Lovatt, C. 2006. Eliminating alternate bearing of the 'Hass' avocado. In: University of California, editor, Proceedings of the California Avocado Research Symposium. University of California, Riverside, CA, USA. p. 127-142.

Lovatt, C., and W. Dugger. 1984. Boron. In: E. Frieden, editor, Biochemistry of the essential ultratrace elements. Vol. 3. Plenum, NY, USA. p. 389-421.

Loupassaki, M., M. Vasilakakis, and S. Androulakis. 1995. The time of flowering of avocado and the female and male opening of flowers in Crete. Adv. Hort. Sci. 9:37-42.

Massey, F.J. 1951. The Kolmogorov-Smirnov test for goodness of fit. J. Am. Stat. Assoc. 46:68-78.

Ramírez-Gil, J. 2016. Tratamientos pregerminativos y masa de la semilla como estrategia para mejorar la producción de plántulas de aguacate raza guatemalteca. Cultivos Trop. 37(4):115-125.

Ramírez-Gil, J., D. Castañeda, y J. Morales. 2014. Alternativas microbiológicas para el manejo de Phytophthora cinnamomi Rands., en Persea americana Mill. bajo condiciones de casa-malla. Cultivos Trop. 35(4):19-27. 
Ramírez-Gil, J., D. Castañeda, and J. Morales. 2017. Production of avocado trees infected with Phytophthora cinnamomi under different management regimes. Plant Pathol. 66:623-632. doi:10.1111/ppa.12620.

Ramírez-Gil, J.G., L. Osorno, J.G. Morales, and N.W. Osorio. 2013. Addition of glucose as carbon source improves the Glomus Fasciculatum-Trichoderma harzianum-Leucaena leucocephala interactions. In: Mexican Society for Biotechnology and Bioengineering (SMBB), editors, Proceedings of the XV National Congress of Biotechnology and Bioengineering and $12^{\text {th }}$ International Symposium on the Genetics of Industrial Microorganisms (GIM). SMBB, Cancun, QR, MEX. p. 1-2.

R-Core-Team. 2013. R: A language and environment for statistical computing. R Foundation for Statistical Computing, Vienna, AUT.

Rebolledo, A., y S. Romero. 2011. Avances en investigación sobre el comportamiento productivo del aguacate (Persea americana Mill.) bajo condiciones subtropicales. Corpoica Cienc. Tecnol. Agropecu. 12:113-120. doi:10.21930/rcta. vol12_num2_art:220

Romero, M.A. 2011. Comportamiento fisiológico del aguacate (Persea americana Mill.) variedad Lorena en la zona de Mariquita, Tolima. Tesis M.Sc., Universidad Nacional de Colombia, Bogotá, COL.

Rossouw, T., and P. Robbertse. 2001. Effect of gibberellic acid treatments on flower development of avocado. SAAGA. 24:1-2.

Scholefield, P., M. Sedgley, and D. Alexaner. 1985. Carbohydrate cyling in relation to shoot growth, floral initiation and development and yield in the avocado. Sci. Hort. 25:99-110.

Salazar-García, S., E. Lord, and C. Lovatt. 1998. Inflorescence and flower development of the 'Hass' avocado during “on” and “off” crop years. J. Amer. Soc. Hort. Sci. 123:537-544.

Salazar-García, S., and C. Lovatt. 2000. Use of GA3 to manipulate flowering and yield of 'Hass' avocado. J. Amer. Soc. Hort. Sci. 125:25-30.

Salazar-García, S., and C. Lovatt. 2002. Flowering of avocado (Persea Americana Mill.). I. Inflorescence and flower development. Rev. Chapingo Serie Hort. 8:71-75.

Tomer, E., and M. Gottreich. 1978. Abnormalities in avocado (Persea americana Mill.) ovule development. Bot. Gazette 139:81-86.

Wilkie, J., M. Sedgley, and T. Olesen. 2008. Regulation of floral initiation in horticultural trees. J. Exp. Bot. 59:3215-3228.

Whiley, A., C. Winks, R. Stephenson, and E. Winston, 1988. Boron nutrition of subtropical and tropical fruit and nut crops in Queens. Maroochy Hort. Res. Station Report. 5:103-104.

Webster, A., and J. Spencer. 1999. New strategies for the chemical thinning of apple (Malus domestica Borkh.) cultivars Queen cox and Royal Gala. J. Hort. Sci. Biotechnol. 74:337-346.

Wolstenholme, B. 1986. Energy costs of fruiting as a yield-limiting factor with special reference to avocado. Acta Hort. 175:121-126.

Wolstenholme, B. 1987. Theoretical and applied aspects of avocado yield as affected by energy budgets and carbon partitioning. SAAGA 10:58-61.

Wolstenholme, B.N. 2010. Alternate bearing in avocado: an overview. www.avocadosource.com/papers/southafrica_papers/ wolstenholmenigel2010.pdf. (accessed 16 set. 2016).

Zilkah, S., I. Klein, S. Feigenbaum, and S. Weinbaum. 1987. Translocation of foliar applied urea ${ }^{15} \mathrm{~N}$ to reproductive and vegetative sinks of avocado and its effect on initial fruit set. J. Amer. Soc. Sci. 112:1061-1065. 